\title{
DESIGN AND TESTING OF THE NEW ION CHAMBER LOSS MONITOR FOR SNS
}

\author{
R. L. Witkover, TechSource, Inc., Santa Fe, NM \\ D. Gassner, Brookhaven National Laboratory, Upton, NY 11973
}

\begin{abstract}
An ion chamber beam loss monitor has been designed for the Spallation Neutron Source (SNS). The new detector overcomes concerns with the original design about slow ion collection times and low collection efficiency at high loss rates. Prototypes have been built and tested with static sources and pulsed beams. Commercially made preproduction units have been fabricated and laboratory tested. Details of the design and test results will be presented.
\end{abstract}

\section{BACKGROUND}

Ion chambers (IC's) are the detector of choice at many accelerators for measuring beam loss. Their stability, sample uniformity, flat response over a wide voltage range and sensitivity are strong features. Shafer designed a simple chamber for the Tevatron in 1982[1], which were also used in RHIC at BNL[2]. These have a coaxial 1/4" OD tube inner and 1.5" ID nickel foil outer electrodes in an argon filled glass tube. Testing of the RHIC ICs showed wide sample variations with the preferred bias polarity (electrons collected on the inner electrode). However, when biased with the opposite polarity, unit-tounit variations were small. Due to schedule demands the non-preferred polarity was used in RHIC. The resulting slower ion signal rise time and earlier saturation were not a problem in RHIC, but would be important concerns in SNS. Recently it was found that a crimp in the outer electrode at the support attachment was the cause of the problem and was eliminated by a simple fix. It is likely that subtle changes in the fabrication technique over the intervening 15 years caused the problem. Tests on 8 samples built with this change showed good reproducibility[3].

The SNS will deliver $10^{14}$ protons to a liquid mercury target at a rate of $60 \mathrm{~Hz}$. The $1 \mathrm{msec}$ long $\mathrm{H}$-minus pulse will be accelerated through the Linac to $1 \mathrm{GeV}$. It then is transported in the HEBT to the Ring where it is accumulated in a single 695 nsec proton bunch over approximately 1000 turns. The beam is then extracted and sent down the RTBT line to the target. The Beam Loss Monitor (BLM) system will utilize nearly 300 ICs along the beam path. In the Ring and RTBT the detectors must operate over an intensity range of $10^{3}$. The dose rate in RTBT even from low percentage losses could be significant due to the short pulse length and high

\footnotetext{
* Work performed under the auspices of the U.S.Dept. of Energy.
}

intensity. While the ICs do not need to resolve the time structure of the bunch (PMTs will be used for that) they must be able to provide a fast beam abort signal to the Machine Protection System (MPS). Subsequent analysis and tests showed that the FNAL ICs, even with the fix, would not satisfy the signal rise time and high dose rate requirements. A new design was needed.

\section{RISE TIME}

While electrons are collected in a few microseconds the heavier ions take much longer. At $2000 \mathrm{~V}$ the ion collection time for the FNAL IC would be close to 700 $\mu$ sec. For a step loss the ion portion of the signal would rise over the $1 \mathrm{msec}$, and fall with a $700 \mu \mathrm{sec}$ tail. While the electron signal would allow a rapid beam abort, the waveform during the pulse would require unfolding.

The positive ion transit time is given by:

$$
t=\frac{d^{2}}{\mu_{0} V\left(P_{0} / P\right)}
$$

where:

$\mu_{0}=$ Ion mobility at STP $\left[\mathrm{cm}^{2} /(\mathrm{V}-\mathrm{sec})\right]$

$\mathrm{V}=$ Applied Voltage $[\mathrm{V}]$

$\mathrm{P}_{0}=$ Atmospheric pressure

$\mathrm{P}=$ Working pressure

$\mathrm{d}=$ Effective electrode separation $[\mathrm{cm}]$ for cylindrical geometry[4]

The transit time can be reduced by decreasing the electrode gap, but the chamber must be lengthened to keep the same sensitivity[5]. By increasing the inner radius to 0.5 " the ion collection time at $3 \mathrm{kV}$ bias would be reduced from $560 \mu \mathrm{sec}$ to $72 \mu \mathrm{sec}$.

\section{COLLECTION EFFICIENCY}

Thomson derived the equations for the collection efficiency in 1899 , but they have only been solved for

$$
d=\left[\left(a^{2}-b^{2}\right) \frac{\ln (a / b)}{2}\right]^{1 / 2}
$$

limited ranges. Using the "equivalent" gap ("d" above), the parallel plate solutions can be applied to cylindrical geometry. Boag and Wilson[6] found a solution assuming second and third order polynomials for the ionization distributions, and steady state radiation. Their result, which gives a good fit for efficiency $>0.7$ is given by: 


$$
f=\frac{1}{1+\frac{\xi^{2}}{6}} \quad \text { where: } \quad \xi^{2}=\frac{\alpha}{e k_{1} k_{2}} \frac{P}{P_{0}} \frac{d^{4} q}{V^{2}}
$$

e is the electron charge,

$\alpha$ is the first Townsend recombination coefficient,

$\alpha=1 \times 10^{-6}\left[\mathrm{~cm}^{3} / \mathrm{sec}\right]$ for Argon

$\mathrm{k}_{1}$ is the electron mobility $=1.8\left[\mathrm{~cm}^{2} /(\mathrm{V}-\mathrm{sec})\right]$

$\mathrm{k}_{2}$ is the ion mobility $=1.3\left[\mathrm{~cm}^{2} /(\mathrm{V}-\mathrm{sec})\right]$

$\mathrm{q}$ is the ionization charge density

$$
q=\frac{I_{\text {signal }}}{v} \times 3 \times 10^{9}
$$

where $\mathrm{I}_{\text {signal }}$ is the current generated by beam loss and $\mathrm{v}$ is the active volume of the ion chamber. For the FNAL IC, the sensitivity is $70 \mathrm{nA} / \mathrm{rad} / \mathrm{sec}$, for a volume of $110 \mathrm{~cm}^{3}$. For a $1 \%$ local loss, the dose rate is $9.2 \mathrm{kRad} / \mathrm{sec}$ during the Linac pulse.

The collection efficiency and ion transit time are shown in Figure 1 as a function of inner cylinder radius.

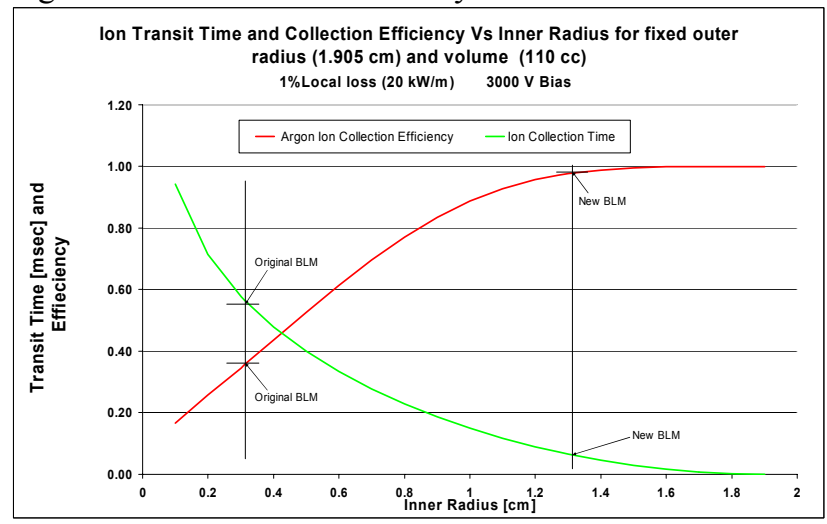

Figure 1: Ion transit time and collection efficiency

This calculation is for continuous radiation, but Boag[7] also found a solution for the pulsed radiation when the loss occurs in a time short compared with the ion transit time. The application is not clear in the Linac and Ring where the pulse length is comparable to the ion transit time for the FNAL chamber. However for the RTBT pulse for a $1 \%$ local the calculation gives the efficiency as $21 \%$ for the FNAL IC and $60 \%$ for the new design.

\section{THE NEW ION CHAMBER DESIGN}

Clearly the new design is superior on paper, but careful high voltage design would be required due to the smaller gap. Guard electrodes were used to divert leakage from the HV electrode to ground and define the active region. Voltage gradients have been reduced by rounding the electrode ends. Figure 2 shows the new design. Two prototypes were tested at BNL. The first was filled with Nitrogen at 1 ATM. A second prototype with a higher voltage feedthrough and improved ceramic and guard rings was able to hold $4.5 \mathrm{kV}$ with 1 ATM Argon.

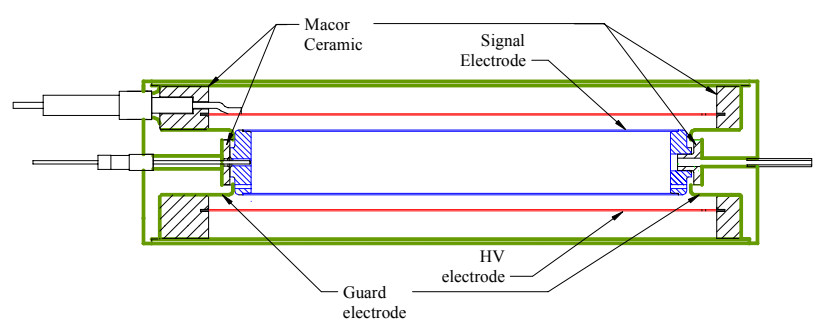

Figure 2: Design of new SNS ion chamber.

\section{TESTS WITH BEAM}

An FNAL IC and Prototype 1 were installed after the BNL $200 \mathrm{MeV}$ Linac. Figure 3 shows the results. The larger FNAL IC signals were due to the difference in proximity to the loss and the use of Argon versus Nitrogen. The wide scatter of points was probably due to beam motion since the beam current did not show this variation. The reason positive bias is not preferred is clear. Prototype 1 shows fairly flat response.

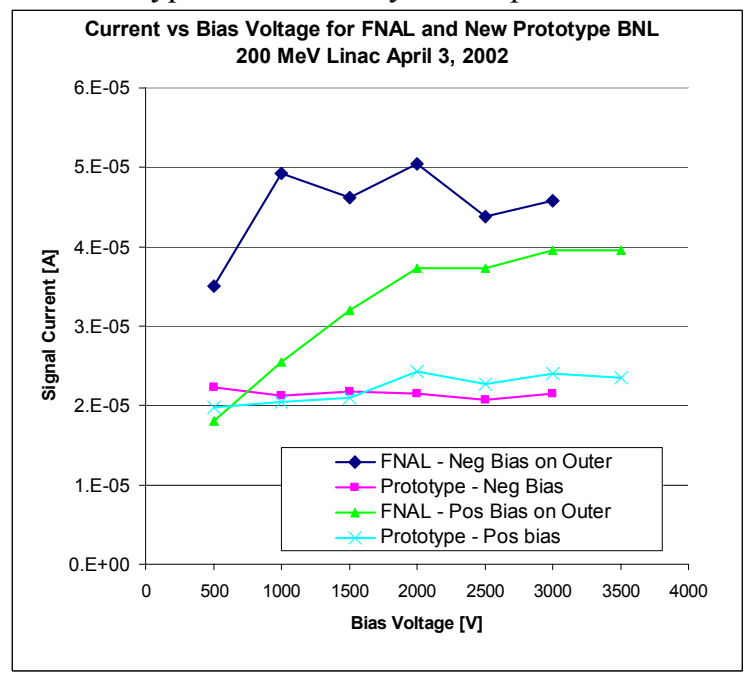

Figure 3: Response of FNAL IC and SNS prototype IC to $200 \mathrm{MeV}$ Linac beam. Approximately $1000 \mathrm{~W} / \mathrm{m}$ loss.

Figures $4 \mathrm{a}$ and $4 \mathrm{~b}$ show the results using the prototype analog front end (AFE) circuitry designed for SNS[8] The first stage is a current amplifier with a $35 \mathrm{kHz} \mathrm{BW}$ using a Burr-Brown OPA627BM. The slow ion transit time can be clearly seen after the pulse in Fig 4a. This also causes the gradual rise during the pulse. The "noise" during the pulse is modulation of the beam to tailor the stacking in the Booster. The signal from the Prototype ion chamber has a much faster ion collection time, as expected and does not show the rise during the pulse. 


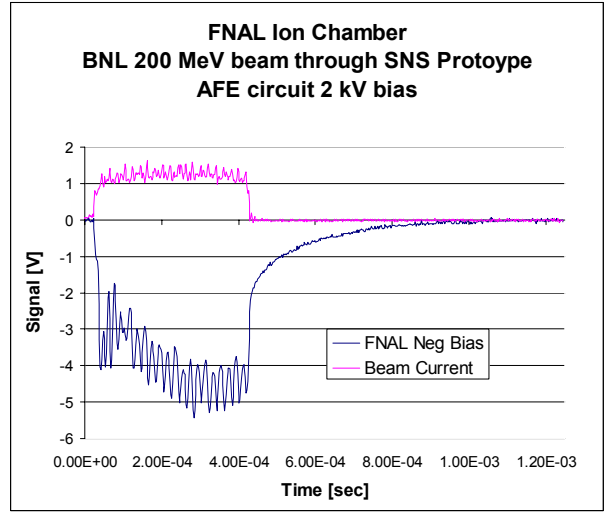

Figure 4a: FNAL IC BNL Linac Beam Test.

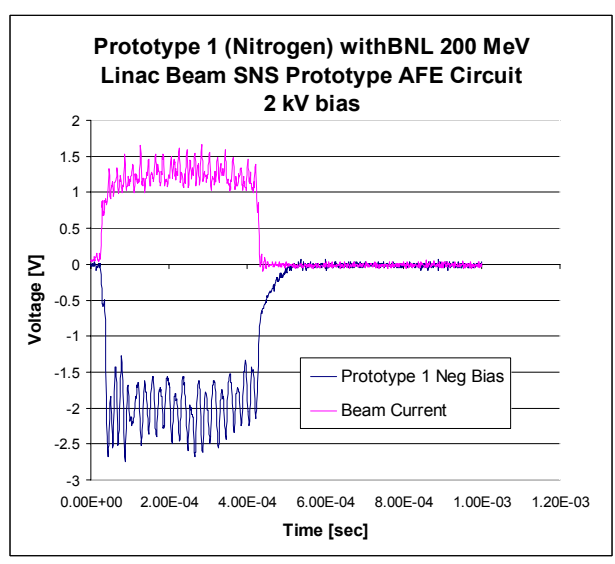

Figure 4b: SNS Prototype IC Linac Beam Test

\section{STATUS}

LND Inc.[9] has produced 10 units of the new design based on BNL supplied drawings. Tests indicate that all units reached $5 \mathrm{kV}$ without breakdown and appeared to give the expected sensitivity. A typical calibration scan is shown in Figure 5.

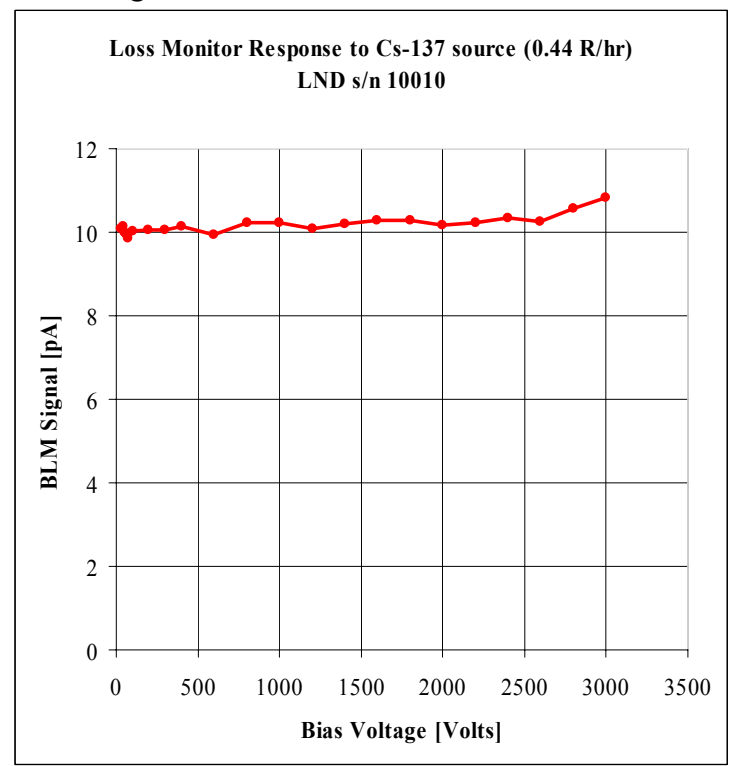

Figure 5: Response of typical new ion chamber.

\section{CONCLUSIONS}

A new design ion chamber has been developed for the SNS application which provides significantly faster ion transit times and can tolerate higher dose rate losses than the FNAL IC. Tests with beam confirm the calculations. In addition, the all ceramic and metal construction is likely to be less susceptible to breakage than the glass FNAL chambers. However, the new chambers will be about twice the cost of the FNAL design and may not be justified in all applications. A pre-production run has indicated that they can be successfully manufactured.

\section{ACKNOWLEDGEMENTS}

The authors wish to acknowledge the contributions of C. J. Liaw in the mechanical design, Dave Kipp is assembling the prototype chambers and Paul Ziminski in testing the chambers with the cesium source. The prototype circuit used in the beam tests was built and tested by Chaofeng Mi. Anthony Curcio (BNL) was invaluable in the installation and setup of the beam tests.

\section{REFERENCES}

[1] R. E. Shafer, et al., The Tevatron Beam Position and Beam Loss Monitoring Systems", Proc. 12 $2^{\text {th }}$ Int'l Conf on High Energy Accel., FNAL (1983) p609

[2] Witkover, R. L., Michnoff, R. J., Geller, J. M., "RHIC Beam Loss Monitor System Initial Operation", Proceedings of the 1999 PAC, NY, 1999, 2247

[3]Witkover, R. L. and Gassner, D., "Design of an Improved Ion Chamber for the SNS", BIW2002, AIP Conf Proc 648 (2002) p337

[4] Boag, J. W. in "Radiation Dosimetry, Vol. II", F. Attix, et al, eds.,Academic Press (1966), p22

[5] R. Witkover," Proposal for a Quasi-Parallel Plate, Cylindrical Geometry Ion Chamber for SNS", November 7, 2001, unpublished

[6] Boag, J. W. and Wilson, T., "The saturation curve at high radiation intensity", Brit. J. Phys., 3 222-9, (1952) and Loc. Cit (5), p16

[7] Boag, J. W., "the Dosimetry of Ionizing Radiation", Vol. 2, Ed. By K. R. Kase, B. E. Bjamgard and F. H. Attix, Academic Press, (1987), p 191

[8] Witkover, R. L. and Gassner, D., "Preliminary Design of the SNS Beam Loss Monitoring System", BIW2002, AIP Conf Proc 648 (2002) p345

[9] LND, Inc., 3230 Lawson Blvd., Oceanside, NY 11572 